\title{
Partial Anterior Cervical Cord Infarction Following Vertebral Artery Dissection
}

\author{
M. Machnowska, F. Moien-Afshari, C. Voll, S. Wiebe
}

Can. J. Neurol. Sci. 2008; 35: 674-677

Infarction of the cervical spinal cord is less common than thoracolumbar cord infarction and accounts for 7-25\% of cord infarcts. ${ }^{1,2}$ Causes include venous and arterial thromboses, vasculitis, fibrocartilaginous embolism, cocaine misuse, epidural anaesthesia, systemic hypotension, arteriosclerosis, Caisson disease, spinal tumor, arteriovenous malformations (AVMs) and arterial dissections. ${ }^{3}$ An increasingly recognized cause for cervical spinal cord infarction is vertebral artery dissection. Twelve reported cases of unilateral or bilateral vertebral artery dissection causing ischemia of the cervical cord were found in a review of literature from 1989-2007. ${ }^{4-14}$ While more than $80 \%$ of patients with vertebral artery dissection are reported to develop posterior circulation brain ischemia, spinal cord infarction is a rare manifestation. ${ }^{15}$

The clinical presentations and areas of involvement of the spinal cord are quite variable among these cases. Acute cord infarction may present as localized cervical pain $^{3}$ which may be the cause of diagnostic confusion. In only two of these cases did unilateral vertebral artery dissection cause a clinical anterior cord syndrome..$^{6,7}$ One vertebral artery frequently supplies the dominant feeder to the anterior spinal artery; therefore compromise of one vertebral artery often causes ischemic changes in the cord bilaterally. ${ }^{16}$ Laufs et al, however, described unilateral cervical cord involvement following ipsilateral vertebral artery dissection. ${ }^{14}$

\section{CASE RePort}

A 77-year-old male with a history of hypertension, stable angina, previous tobacco use and a transient ischemic attack eight years prior to this admission presented with acute onset bibrachial weakness and numbness while driving a motor vehicle. These symptoms were preceded for several hours by severe non-radicular cervical pain which was constant, stabbing in nature, and localized to the right side of his neck. Over the ensuing half hour he noted difficulty walking due to weakness of his right lower extremity accompanied by urinary retention. He was admitted to his local hospital and subsequently transferred to our facility on the second day. On initial examination cranial nerve examination was normal. The right upper extremity was plegic and the right lower extremity and left upper extremity were weak (Table 1).

Deep tendon reflexes were reduced in the right upper extremity; the plantar reflexes were bilaterally extensor. Sensory examination revealed a decrease in pin prick and temperature sensation in a collar distribution extending from C3-T5 anteriorly and posteriorly; a bilateral patchy decrease in

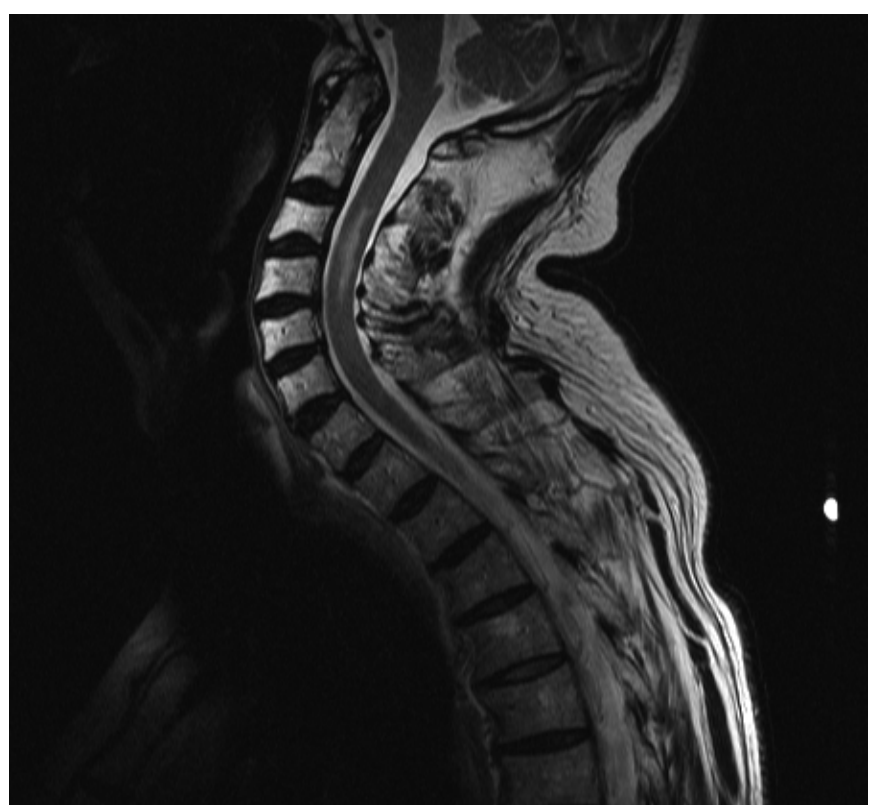

Figure 1. T2W MRI shows hyperintensity in the anterior cord from C3-C4.

vibration from T5 distally. Joint position, graphaesthesia and stereognosis were intact bilaterally. Complete blood count (CBC), erythrocyte sedimentation rate (ESR), electrolytes, fasting glucose, $\mathrm{HbA} 1 \mathrm{C}$, and coagulation tests were unremarkable. Low density lipoprotein (LDL) cholesterol and total cholesterol/ high density lipoprotein (HDL) ratio were high (3.58, and 6.6 respectively). Cortisol and adrenocorticotropic hormone (ACTH) levels were normal. The ECG, cardiac telemetry and cardiac enzymes were normal. An echocardiogram

From the Departments of Neurology and Radiology, Royal University Hospital, 103 Hospital Drive - University of Saskatchewan, Saskatoon, Saskatchewan, Canada. Received November 30, 2007. Final Revisions Submitted May 2, 2008. Correspondence to: M. Machnowska, Departments of Neurology and Radiology Royal University Hospital, 103 Hospital Drive - University of Saskatchewan Saskatoon, Saskatchewan, S7N, 0W8, Canada. 
revealed an ejection fraction of $63 \%$, and no signs of intracardiac thrombus. Serum neuro-myelitis optica IgG antibody assay was negative.

Magnetic resonance imaging (MRI) studies of the brain including diffusion-weighted images were normal. An MRI of the cervical spinal cord showed anterior cord hyperintensity on sagittal T2-weighted images most prominent from $\mathrm{C} 2$ to $\mathrm{C} 5$ (Figure 1). Axial multiple-echo data image combination (MEDIC) images showed hyperintensities in the anterior lateral horns of the spinal cord bilaterally most apparent at the $\mathrm{C} 3-\mathrm{C} 4$ levels (Figure 2). The replacement of the normal "flow void" in the right vertebral artery along its entire course by an isointense density on axial T1 weighted images suggested the presence of acute (deoxyhemoglobin) hematoma in a dissected vessel ${ }^{17}$ (Figure $3 \mathrm{a}, \mathrm{b}$ ). Contrast enhancement, following intravenous gadolinium administration was seen on the sagittal $\mathrm{T} 1$ image situated centrally in the cord from $\mathrm{C} 2-\mathrm{C} 4$.

The patient was anticoagulated with heparin. On the fourth day after initial presentation, the left upper extremity paresis worsened and his left lower extremity also became paretic with strength measurements of $2 / 5$ in the left upper and lower extremity. Repeat 2 dimensional time of flight MR angiography sequences demonstrated apparent occlusion of the entire right vertebral artery. A T1 fat saturation skull base sequence again showed the lack of flow void in the right vertebral artery, now replaced with hyperintense signal in keeping with evolution to the methemoglobin stage of hematoma, supporting the diagnosis of an acute traumatic dissection rather than a chronic occlusion such as intraluminal thrombus, extramural mass, or congenital

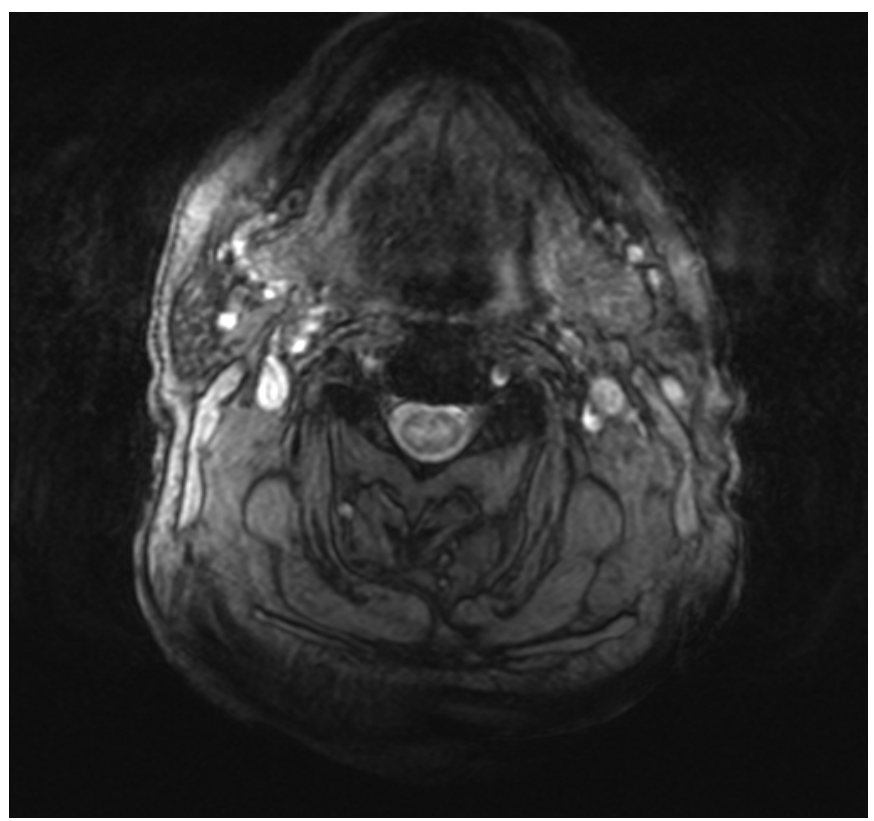

Figure 2. Axial MEDIC images show anterior horn cell hyperintensities at the level of $C 3$.
Table: The Medical Research Council muscle power grading scale

\begin{tabular}{lcc}
\hline & Right & Left \\
Trapezius & 5 & 5 \\
Deltoid & 0 & 0 \\
Triceps & 0 & 4 \\
Biceps & 0 & 3 \\
Wrist extensors & 0 & 5 \\
Wrist flexors & 0 & 5 \\
Finger extensors & 0 & $4+$ \\
Abductor pollicis brevis & 0 & $4+$ \\
First Dorsal Interosseous & 0 & 5 \\
Iliopsoas & 1 & 5 \\
Quadriceps & 5 & 5 \\
Hip adductors & 2 & 5 \\
Hip Abductors & 3 & 5 \\
Tibialis Anterior & 0 & 5 \\
Gastrosoleus & $4+$ & 5 \\
Flexor hallucis Longus & 0 & 5 \\
\hline
\end{tabular}

variation (Figure 3c). Mild swelling of the cord was also noted. A CT angiogram (CTA) performed six days after the onset of symptoms, showed a tortuous right brachiocephalic artery giving rise to the right vertebral artery only minimally filled with contrast at its proximal origin. The majority of the vessel was non-enhancing with a slight amount of enhancement from the level of $\mathrm{C} 2$ to the basilar artery most likely due to retrograde flow.

On the sixth day of admission he developed hypotension, bradycardia and hypothermia consistent with spinal shock and respiratory distress, worse in the recumbent position and accompanied by paradoxical respiration. Repeat MRI showed evolution of infarct in the transverse dimension, most prominent at the C3-C4 level particularly at the right aspect, with no evidence of hemorrhagic change in the lesion.

The patient was transferred to a rehabilitation hospital, where he regained strength on the left, achieving antigravity hip flexion, knee extension, and elbow flexion/extension; gravity eliminated hip abduction and knee flexion and weak ankle dorsiflexion. He did not regain right sided strength. Two weeks after transfer he developed an inferior wall myocardial infarction and, unfortunately, passed away.

\section{DISCUSSION}

Our patient's initial presentation with predominantly right arm paresis, right leg weakness, dissociative sensory loss and nuchal pain was consistent with a partial anterior cord syndrome initially involving C5 to T2 segments but with the subsequent development of paradoxical respiration ${ }^{18}$ following an episode of hypotension consistent with diaphragmatic paralysis, in turn 

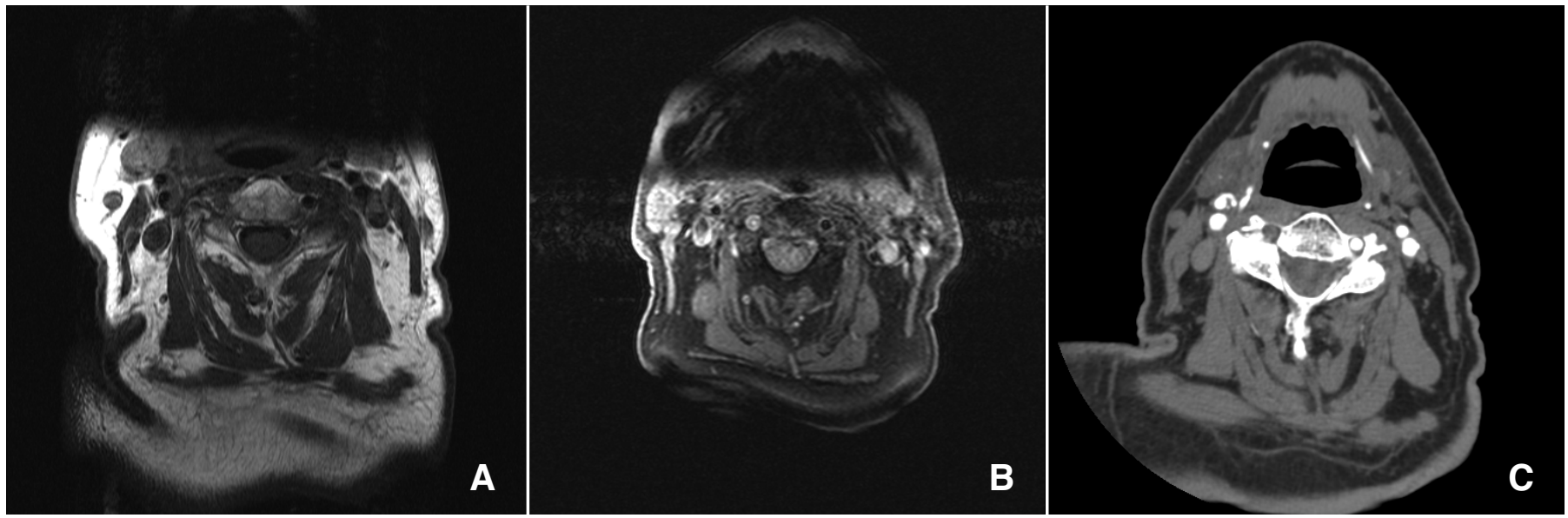

Figure 3. A. CT angiogram showing absence of contrast in the right verteberal artery at the level of C3. B. T1W MRI performed 24 hrs after presentation shows $T 1$ isointensity and lack of flow void in the right vertebral artery in keeping with acute (deoxyhemoglobin) hematoma in the vessel wall. C. T1W MRI performed $4 d$ after presentation shows T1 hyperintensity in keeping with evolving (methemoglobin) hematoma in the vessel wall.

suggesting segmental involvement up to the C3-C5 level ${ }^{19}$. An MRI confirmed changes consistent with clinical localization.

A unique aspect of the presentation was a suspended dissociated sensory loss (only pain and temperature) in the distribution of C3-T5 with preserved sensation above and below. This pattern has been previously described to occur with occlusion of a central sulcal artery. ${ }^{20}$ To our knowledge there is only one report of similar sensory deficits limited to a number of adjacent dermatomes in the anterior spinal artery territory. ${ }^{21}$

Subsequent investigation supported unilateral vertebral artery dissection (VAD) which we considered to be the likely cause of cord infarction. Additional investigation demonstrated no cardioembolic source, hypercoaguable state or inflammatory vascular disorder; in addition there was no history of trauma or disc disease to suggest fibrocartilaginous embolism. We did not proceed with selective spinal angiography since it would have increased risk to the patient without changing management; the findings of hematoma on serial MRI T1 weighted images combined with a finding of complete occlusion on CTA are highly diagnostic of a vertebral artery dissection. ${ }^{22}$

Although vertebral artery dissection is a common cause of brainstem and cerebral ischemia, it is an unusual cause of cord infarction. In a series of 111 patients with vertebral artery dissection reported by Crum only two presented with spinal manifestations: one with a C5-C6 radiculopathy and the other with a cervical myelopathy. ${ }^{23}$ Common manifestations include in order of frequency, bilateral ischemic myelopathy, cervical radiculopathy, often at $\mathrm{C} 5-\mathrm{C} 6$ and primarily motor and chest pain $^{23}$ and neck pain. ${ }^{11}$

Abnormalities of ventilation have been reported previously in a patient with combined brainstem and cord infarction secondary to VAD. ${ }^{24}$ Brown Sequard syndrome is reported uncommonly in association with cervical cord infarction complicating VAD. ${ }^{9}$ Bilateral upper extremity amyoatrophy ${ }^{6}$ and posterior spinal cord infarction $^{8}$ has rarely been described. Causes range from spontaneous dissection, minor trauma, or hypotension complicating circulatory arrest.

An interesting aspect of our patient's presentation was the initial relative sparing of left-sided function. The ventral $2 / 3$ of the cervical spinal cord typically receives its blood supply from the anterior spinal artery (ASA). The origin of the main feeders of the cervical part of the anterior spinal artery is highly variable but usually is the vertebral arteries on one or both sides. There are however, many deviations from this pattern, resulting in variable clinical deficits with cord infarction. In one such variation, parallel branches from the vertebral arteries course over several segments and do not fuse with the anterior spinal artery, but instead terminate as a larger central artery. ${ }^{16}$ In these instances, the ASA typically receives its supply predominantly from one vertebral artery. Turnbull showed that in cases where the ASA is duplicated, each vessel gives off sulco-commissural arteries supplying the ipsilateral half of the cord..$^{25} \mathrm{~A}$ similar vascular anomaly in the supply of the central cervical cord in our patient would have resulted in the relative initial sparing of left sided function. Left sided involvement was seen only after evolution of the infarct on the fourth day of admission, possibly due to bilateral anterior horn cell involvement because of watershed infarction.

While treatment of vertebral artery dissection remains controversial due to the lack of controlled clinical trials, early anticoagulation is thought to decrease the risk of distal thrombosis of the vertebral artery and to decrease the risk of posterior circulation infarction. ${ }^{22}$ Given the absence of contraindications to anticoagulation, such as intracranial extension of the dissection, a large infarct or hemorrhagic transfer of the existing infraction, the patient was anticoagulated with unfractionated heparin for one week and started on a therapeutic dose of warfarin. ${ }^{26}$ The patient was transferred to a 
rehabilitation hospital following discharge where he began to regain left sided function; furthering our hypothesis that loss of left sided function was likely due to a watershed infarction on the fourth day of admission.

\section{REFERENCES}

1. Masson C, Pruvo JP, Meder JF, Cordonnier C, Touze E, De La Sayette V, et al. Spinal cord infarction: Clinical and magnetic resonance imaging findings and short term outcome. J Neurol Neurosurg Psychiatry. 2004; 75(10):1431-5.

2. Cheshire WP, Santos CC, Massey EW, Howard JF, Jr. Spinal cord infarction: Etiology and outcome. Neurology. 1996; 47(2): $321-30$.

3. von Kummer R, Back T, editors. Magnetic Resonance Imaging in Ischemic Stroke. Berlin: Springer, 2006.

4. Pryse-Phillips W. Infarction of the medulla and cervical cord after fitness exercises. Stroke. 1989; 20(2):292-4.

5. Gutowski NJ, Murphy RP, Beale DJ. Unilateral upper cervical posterior spinal artery syndrome following sneezing. J Neurol Neurosurg Psychiatry. 1992; 55(9):841-3.

6. Pullicino P. Bilateral distal upper limb amyotrophy and watershed infarcts from vertebral dissection. Stroke. 1994; 25(9):1870-2.

7. Suh DC, Kim SJ, Jung SM, Park MS, Lee JH, Rhim SC. MRI in presumed cervical anterior spinal artery territory infarcts. Neuroradiology. 1996; 38(1):56-8.

8. Bergqvist CA, Goldberg HI, Thorarensen O, Bird SJ. Posterior cervical spinal cord infarction following vertebral artery dissection. Neurology. 1997; 48(4):1112-5.

9. Goldsmith P, Rowe D, Jager R, Kapoor R. Focal vertebral artery dissection causing brown-sequard's syndrome. J Neurol Neurosurg Psychiatry. 1998; 64(3):415-6.

10. Berg D, Mullges W, Koltzenburg M, Bendszus M, Reiners K. Manin-the-barrel syndrome caused by cervical spinal cord infarction. Acta Neurol Scand. 1998; 97(6):417-9.

11. Hundsberger T, Thomke F, Hopf HC, Fitzek C. Symmetrical infarction of the cervical spinal cord due to spontaneous bilateral vertebral artery dissection. Stroke. 1998; 29(8):1742.

12. De la Sayette V, Leproux F, Letellier P. Cervical cord and dorsal medullary infarction presenting with retro-orbital pain. Neurology. 1999; 53(3):632-4.
13. Weidauer S, Claus D, Gartenschlager M. Spinal sulcal artery syndrome due to spontaneous bilateral vertebral artery dissection. J Neurol Neurosurg Psychiatry. 1999; 67(4):550-1.

14. Laufs H, Weidauer S, Heller C, Lorenz M, Neumann-Haefelin T. Hemi-spinal cord infarction due to vertebral artery dissection in congenital afibrinogenemia. Neurology. 2004; 63(8):1522-3.

15. Arnold M, Bousser MG. Clinical manifestations of vertebral artery dissection. Front Neurol Neurosci. 2005; 20: 77-86.

16. Thron AK. Vascular Anatomy of the Spinal Cord: Neuroradiological Investigations and Clinical Syndromes. Wien: Springer-Verlag, c1988.

17. Shah GV, Quint DJ, Trobe JD. Magnetic resonance imaging of suspected cervicocranial arterial dissections. J Neuroophthalmol. 2004; 24(4):315-8

18. Wilcox PG, Pardy RL. Diaphragmatic weakness and paralysis. Lung. 1989; 167(6):323-41.

19. Howard RS, Thorpe J, Barker R, Revesz T, Hirsch N, Miller D, et al. Respiratory insufficiency due to high anterior cervical cord infarction. J Neurol Neurosurg Psychiatry. 1998; 64(3):358-61.

20. Bradley WG, Daroff RB, Fenichel GM, Jankovic J, editors. Neurology in Clinical Practice. Philadelphia: Elsevier, 2004.

21. Casselman JW, Jolie E, Dehaene I, Meeus L. Gadolinium-enhanced MR imaging of infarction of the anterior spinal cord. AJNR Am J Neuroradiol. 1991; 12(3):561.

22. Flis CM, Jager HR, Sidhu PS. Carotid and vertebral artery dissections: clinical aspects, imaging features and endovascular treatment. Eur Radiol. 2007; 17: 820-34.

23. Crum B, Mokri B, Fulgham J. Spinal manifestations of vertebral artery dissection. Neurology. 2000; 55(2):304-6.

24. Lanczik O, Szabo K, Lecei O, Binder J, Thiel S, Gass A, et al. Central respiratory dysfunction following vertebral artery dissection. Neurology. 2006; 66(6):944.

25. Turnbull IM, Brieg A, Hassler O. Blood supply of cervical spinal cord in man. A microangiographic cadaver study. J Neurosurg. 1966; 24(6):951-5.

26. Schievink, W. The treatment of spontaneous carotid and vertebral artery dissections. Curr Opin in Cardiol (2000) 15: 316-21. 